medRxiv preprint doi: https://doi.org/10.1101/2021.04.28.21256242; this version posted June 24, 2021. The copyright holder for this preprint (which was not certified by peer review) is the author/funder, who has granted medRxiv a license to display the preprint in perpetuity.

It is made available under a CC-BY 4.0 International license.

Title: SARS-CoV-2 seroprevalence and gender-related haematological features in asymptomatic blood donors

Running Title: SARS-CoV-2 seroprevalence and gender-related haematological features

M Carmen Martín ${ }^{a}$, M Isabel González a , Nuria Holgado a , Ana I Jimenez a , Nuria Ortega $^{a}$, Isabel Page ${ }^{a}$, Alba Parrado ${ }^{a}$, María Pérez ${ }^{a}$, Lydia Blanco-Peris ${ }^{a}$, a Centro de Hemoterapia y Hemodonación de Castilla y León, Paseo de Filipinos s/n, 47007 Valladolid, Spain

Corresponding author: M Carmen Martín; cmartinalo@saludcastillayleon.es; Centro de Hemoterapia y Hemodonación de Castilla y León, Paseo de Filipinos s/n, 47007 Valladolid, Spain 


\section{SARS-CoV-2 seroprevalence and gender-related hematological features in asymptomatic blood donors}

\section{Abstract}

Background and objectives: COVID-19 can either cause death or go unnoticed but antibodies will remain protecting us of SARS-CoV-2 reinfection for an uncertain time and to an uncertain extent. Our aim was to describe seroprevalence evolution from summer 2019 to autumn 2020 in Spain and to describe its relationship with age, blood group and haematological parameters.

Materials and methods: Sera and plasma from historical donation archives excluding convalescent were randomized and a total of 12,313 donations tested by a Chemiluminiscent analysis for anti SARS-CoV-2 N protein total immunoglobulins. Blood donors were $60.9 \%$ males, average age $46+/-13$. Sex, age, blood group, blood cell counts and percentages and immunoglobulin concentrations were extracted from electronic recordings.

Results: A seroprevalence of $6.7 \%$ in blood donors was found by the end of the first wave. No differences by sex, age or blood group were found regarding antibodies. Leukocyte count $(p=0.026)$, haematocrit $(p<0.001)$ and haemoglobin $(p<0.001)$ were lower in positive donations than in negative ones. Sex differences were present in neutrophils, leukocytes, haemoglobin and haematocrit as related to SARS-CoV-2 antibodies.

Conclusions: Seroprevalence due to asymptomatic cases would resemble that of global population. Sex and age would not affect COVID- 
medRxiv preprint doi: https://doi.org/10.1101/2021.04.28.21256242; this version posted June 24, 2021. The copyright holder for this preprint (which was not certified by peer review) is the author/funder, who has granted medRxiv a license to display the preprint in perpetuity.

It is made available under a CC-BY 4.0 International license .

19 susceptibility but its severity. Gender differences related to COVID-19 in leukocytes, haemoglobin and haematocrit would be present in asymptomatic individuals. Further studies are needed to confirm these gender differences as they can help better understand the immune response to COVID-19, its pathogenesis and prognosis.

Keywords: COVID-19, antibodies, immunity, seroprevalence, SARS-CoV-

2

1 Introduction

2 An unknown amount of individuals infected with SARS-CoV-2 present no

3 symptoms or a mild disease that can go unnoticed. This make it difficult to

4 estimate infection rates, prevalence, calculating absolute risks of COVID-

519 or predicting the spread of the virus and the growth of herd immunity.

6 [1]. Immunization either due to infection or to vaccination leads specific $T$

7 cell responses and production of antibodies against SARS-CoV-2 usually

8 peaking 8-15 days after infection [2]. Antibodies that neutralize the virus

9 are the primary protection against COVID-19.

10 Antibody assays are quite different: they either detect antibodies against

11 different viral proteins (S1, S1/S2, RBD or NC) or different immunoglobulin

12 classes: $\lg G, \lg M, \lg A$ or their combinations. It should be noticed that

13 every approved vaccine in Europe to date (May 2020) enhances anti-S

14 responses, whereas an infection leads to a multi specific response to

15 SARS-CoV2 antigens. To make it even messier, many factors can

16 influence test performance, including cross-reactivity with other

17 coronaviruses or platform (laboratory-based vs point-of-care, lateral flow). 
medRxiv preprint doi: https://doi.org/10.1101/2021.04.28.21256242; this version posted June 24, 2021. The copyright holder for this preprint (which was not certified by peer review) is the author/funder, who has granted medRxiv a license to display the preprint in perpetuity.

It is made available under a CC-BY 4.0 International license .

18 Chemiluminiscence assays have suitable performances regarding both sensibility and sensitivity while correlation to neutralizing antibodies would

20 be around 0.7 [3].

21 Our region, Castilla y Leon, with a population of 2.299.598 inhabitants, has

22 accumulated nearly 225.000 COVID-19 confirmed cases

23 (https://analisis.datosabiertos.jcyl.es/), with an overall case-fatality rate of

243.01 to date. Our institution, Centro de Hemoterapia de Castilla y León

25 keeps within its Biobank plasma samples from every single donation along

26 the last ten years, that makes up around one million samples. Any

27 demographic data such as sex or age and laboratory parameters (blood

28 counts, blood group, haemoglobin, etc....) is as well kept in a database

29 (Hemasoft, eDelphyn).

30 Blood donor-based serosurveillance is a powerful and cost-effective

31 strategy to monitor infectious diseases. There are quite a lot of infections

32 for which routine donor screening is undoubtedly useful, including human

33 immunodeficiency virus (HIV), hepatitis C virus (HCV), hepatitis B virus

34 (HBV), human T-lymphotrophic virus (HTLV), and West Nile virus (WNV)

35 [1], babesiosis or Crimea-Congo fever. The use of blood donor samples

36 means we are sampling mainly asymptomatic and recovered cases of

37 COVID-19 (donation is allowed after 28 days following COVID-19

38 resolution of symptoms).

39 Seroprevalence surveys are then needed to monitor coronavirus

40 pandemic, both before and after vaccination strategies [4]. Reported

41 COVID-19 cases do not represent the full SARS-CoV-2 disease burden 
medRxiv preprint doi: https://doi.org/10.1101/2021.04.28.21256242; this version posted June 24, 2021. The copyright holder for this preprint (which was not certified by peer review) is the author/funder, who has granted medRxiv a license to display the preprint in perpetuity.

It is made available under a CC-BY 4.0 International license.

42 due to low detection rates, especially at the beginning of pandemics. Case

43 reports are dependent on patients' seeking health care, massive local

44 screenings or regional tracking activities. Analysis of data from

45 seroprevalence and serosurveillance is a common strategy for estimating

46 underreporting and real disease burden. Time between infection and

47 antibody development or antibody waning must be considered to

48 understand seroprevalence surveys avoiding biases. Serological surveys

49 with a random sampling design of the general population are difficult to

50 perform in a pandemic, but they would yield a seroprevalence estimate

51 least likely to be affected by selection bias. Blood donors are a quite

52 representative subset of general population aged 18-65 and our historical

53 plasma collection was big enough to perform a systematic time-layered

54 randomization.

55 Heterogeneity of susceptibility and transmission are hard to evaluate [5]. A

56 portion of the population is not susceptible to infection from the first

57 pathogen contact. Some of them may have pre-existing immunity via

58 cross-reactivity or particular host factors such as mucosal immunity or

59 trained innate immunity protection (as it has been reported to be conferred

60 by DTP or BCG vaccination)[6]. There is as well a proportion of

61 seronegative individuals that will develop immunity by $\mathrm{T}$ cell mediated

62 responses without exhibiting an antibody response [7].

63 Our starting hypothesis was the existence of a certain number of 64 asymptomatic carriers of the SARS-CoV-2 virus that would develop 65 antibodies against it [7] and subsequently contribute to herd immunity. 
medRxiv preprint doi: https://doi.org/10.1101/2021.04.28.21256242; this version posted June 24, 2021. The copyright holder for this preprint (which was not certified by peer review) is the author/funder, who has granted medRxiv a license to display the preprint in perpetuity.

It is made available under a CC-BY 4.0 International license .

66 Those asymptomatic cases were unnoticeable before SARS-CoV-2 testing

67 began and might had been circulating from an unknown moment. Early cases have been described in France [8], arising the question of whether the pathogen could have been circulating before the official recording of

70 the first cases.

71 The main aim of this study was to determine which percentage of the 72 population has had contact with SARS-CoV-2 at different times and had 73 therefore developed antibodies against it. A secondary goal was to 74 describe related haematological features of seropositive donors and 75 establish whether sex, age, blood group or haematological abnormalities

76 could be related to past COVID-19 infection.

77 Knowledge of COVID-19 epidemiological and haematological features 78 should help make a good forecast of resources for possible future 79 outbreaks or vaccination strategies, and would facilitate decisions in the 80 social sphere by a more reliable estimate of the percentage of immunized 81 individuals.

\section{MATERIAL and METHODS}

83 Seroprevalence and haematological features were studied blood, plasma 84 (excluding convalescent) and platelet donations from July 2019 to October 85 2020. The 101,183 donations from 70,181 donors collected since $8619 / 07 / 2019$ (week -24) to 19/10/2020 (week 43) were systematically 87 randomized to select a minimum of 127 donations per week, calculated on 88 the basis of the total number of donations and the length of the analysis 89 period. Then, 1650 convalescent plasma donation were excluded. A total 
medRxiv preprint doi: https://doi.org/10.1101/2021.04.28.21256242; this version posted June 24, 2021. The copyright holder for this preprint (which was not certified by peer review) is the author/funder, who has granted medRxiv a license to display the preprint in perpetuity.

It is made available under a CC-BY 4.0 International license.

90 of 12,718 samples of 12,313 donations from 11,444 donors over 18 years

91 old were included.

92 All haematological and demographic data were extracted from our

93 electronic database eDelphyn (Hemasoft). Variables analysed included

94 age, sex, blood group, and laboratory data: leukocyte (WBC), neutrophil,

95 lymphocyte, platelet, monocyte, eosinophil and basophil counts

$96\left(\right.$ cells* $\left.10^{3} / \mu \mathrm{L}\right)$ and their percentages, serum immunoglobulins $\lg \mathrm{G}, \lg \mathrm{A}$ and

$97 \lg \mathrm{M}(\mathrm{mg} / \mathrm{dL})$, haemoglobin $(\mathrm{Hb})$, and haematocrit $(\mathrm{HCT})$ were analysed as

98 well.

99 An automated chemiluminiscence double-antigen sandwich immunoassay

100 for the in vitro semi quantitative detection of total antibodies to SARS-CoV-

1012 in human plasma and serum frozen samples. The target antigen of this

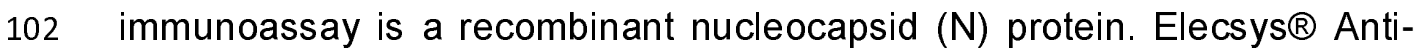

103 SARS-CoV-2 (Roche, Basel, Switzerland) detects antibodies correlating

104 with virus-neutralizing ones and is therefore useful to help characterize the

105 immune reaction to SARS-CoV-2 [9, 10]. Immunoassay was validated by

106 our serology lab by testing of 6 pairs of samples (plasma EDTA and

107 serum) from diagnosed PCR-positive, symptomatic cases infected by mid-

108 April, that were previously reported positive by the Spanish National

109 Microbiology Centre, and checked to be as well positive for IgG

110 (Chemiluminiscence, $\mathrm{N}$ protein, Abbott Alinity S, Chicago USA) and IgA

111 (ELISA, S protein, Euroimmunn, Lübeck, Germany) antiSARS-CoV-2.

112 Another set of ten prepandemic samples, therefore supposed to be

113 negative, were equally analysed. 405 donations were analysed both in

114 serum and plasma to verify interchangeability. A $100 \%$ concordance was 
medRxiv preprint doi: https://doi.org/10.1101/2021.04.28.21256242; this version posted June 24, 2021. The copyright holder for this preprint (which was not certified by peer review) is the author/funder, who has granted medRxiv a license to display the preprint in perpetuity.

It is made available under a CC-BY 4.0 International license.

115 yielded by all these validation assays. The cut-off was that recommended

116 by manufacturer (OD>1 to report reactivity). Researchers performing anti

117 SARS-CoV-2 analyses were blind to the condition of COVID-19

118 convalescence and to any other characteristic of the donors or to the

119 donation dates.

120 Demographic and clinical characteristics of patients are expressed as their

121 mean, median, standard deviation (SD) and interquartile range (IQR) for

122 continuous variables and frequency distributions are reported for

123 categorical variables. Age was analysed both as continuous and

124 categorical variable; in the latter case was recoded into 4 groups: $<30,30-$

$12545,45-60$ and 60-75 years old.

126 Kolmogorov-Smirnov test was performed on each continuous variable to

127 contrast normality. Only $\lg G$ and $\lg M$ serum levels followed normal

128 distributions, therefore non-parametric Mann-Whitney $U$ test was

129 performed. To contrast independence of categorical variables, Pearson's

130 Chi-square and Fisher's exact test were carried out. All tests were

131 calculated with a confidence level of 0.05 .

132 The Biobank is included in the National Registry of Biobanks (RD17 /

133 16/2011) with the number B.0000264. The institution holds an ISO 9001:

1342015 certification endorsing our granting of safety and traceability of any

135 human biological sample we distribute, always behaving Spanish and

136 European rules on human samples and data protection management.

137 This study was conducted according with national regulations, institutional

138 policies and in the tenets of the Helsinki Declaration. It was approved by 
139 the Valladolid Health Area Drug Research Ethics Committee, on June

140 11th, 2020 with the reference number BIO-2020-93. Included donors

141 consented to participate in Biobank research activities. The privacy rights

142 were always observed.

\section{Results}

144 The 101,183 donations from 70,181 donors along 67 weeks (July 2019 to 145 October 2020) were systematically randomized, discarding convalescent 146 plasma ones and a total of 12,313 donations (either whole blood, 147 plasmapheresis or platelet apheresis) were tested for total anti SARS148 CoV-2 antibodies. Donors were 11,444 individuals, 60.91\% males, aged 149 18-75, average $46+/-13,45.8 \% \mathrm{~A}, 40.3 \% \mathrm{O}, 10.56 \% \mathrm{~B}, 3.31 \% \mathrm{AB}$. 150 Baseline characteristics descriptive analyses are summarized in Table 1. 151 Immunoglobulin results are usually performed only once a year in plasma 152 donors.

\section{Table 1. Baseline characteristics of donations}

\begin{tabular}{lccccc} 
& $\mathrm{n}$ & mean & median & $\mathrm{SD}$ & IQR \\
\hline Age & 12313 & $\mathbf{4 4 , 0}$ & $\mathbf{4 5 , 8}$ & 12,8 & $34,2-54,3$ \\
$\lg \mathrm{M}$ & 199 & $\mathbf{1 0 6 6 , 9}$ & $\mathbf{1 0 3 8 , 0}$ & 217,8 & $910-1185$ \\
$\lg \mathrm{A}$ & 199 & $\mathbf{2 3 6 , 6}$ & $\mathbf{2 2 4 , 0}$ & 100,9 & $162-292$ \\
$\lg \mathrm{M}$ & 199 & $\mathbf{1 1 3 , 0}$ & $\mathbf{1 0 0 , 0}$ & 64,3 & $69-138$ \\
Leukocyte & 10105 & $\mathbf{7 , 4}$ & $\mathbf{7 , 2}$ & 1,8 & $6,1-8,5$ \\
Haemoglobin & 11604 & $\mathbf{1 4 , 9}$ & $\mathbf{1 4 , 9}$ & 1,3 & $14-15,8$ \\
Haematocrit & 11604 & $\mathbf{4 5 , 1}$ & $\mathbf{4 5 , 1}$ & 3,5 & $42,6-47,5$ \\
Platelets & 11603 & $\mathbf{2 4 7 , 9}$ & $\mathbf{2 4 3 , 0}$ & 54,3 & $211-280$
\end{tabular}




$\begin{array}{lccccc}\text { Neutrophil count } & 11603 & \mathbf{4 , 4} & \mathbf{4 , 2} & 1,4 & 3,4-5,2 \\ \text { Neutrophil \% } & 11603 & \mathbf{5 8 , 8} & \mathbf{5 8 , 9} & 7,5 & 53,8-63,9 \\ \text { Lymphocyte count } & 11602 & \mathbf{2 , 3} & \mathbf{2 , 3} & 0,7 & 1,9-2,7 \\ \text { Lymphocyte \% } & 11602 & \mathbf{3 1 , 7} & \mathbf{3 1 , 5} & 6,9 & 27-36,2 \\ \text { Monocyte count } & 11602 & \mathbf{0 , 5} & \mathbf{0 , 5} & 0,1 & 0,4-0,6 \\ \text { Monocyte\% } & 11602 & \mathbf{6 , 5} & \mathbf{6 , 4} & 1,5 & 5,5-7,4 \\ \text { Eosinophil count } & 11603 & \mathbf{0 , 2} & \mathbf{0 , 2} & 0,1 & 0,1-0,2 \\ \text { Eosinophil \% } & 11603 & \mathbf{2 , 5} & \mathbf{2 , 1} & 1,8 & 1,3-3,2 \\ \text { Basophil count } & 11603 & \mathbf{0 , 0} & \mathbf{0 , 0} & 0,0 & 0-0 \\ \text { Basophil \% } & 11603 & \mathbf{0 , 5} & \mathbf{0 , 5} & 0,2 & 0,3-0,6\end{array}$

155 No differences in anti SARS-CoV2 reactivity due to sex, age or blood 156 group of donations were found (Table 2).

157 Table 2. Sex, age range and blood group of positive/negative anti 158 SARS-COV2 donations analysed

\begin{tabular}{lcccc} 
& \multicolumn{2}{c}{ Negative } & \multicolumn{2}{c}{ Positive } \\
& n & $\%$ & n & $\%$ \\
\hline Female & 4531 & $38.1 \%$ & 164 & $40.3 \%$ \\
Male & 7375 & $61.9 \%$ & 243 & $59.7 \%$ \\
$<30$ & 3551 & $29.8 \%$ & 104 & $25.6 \%$ \\
30 to 45 & 5036 & $42.3 \%$ & 180 & $44.2 \%$ \\
45 to 60 & 1151 & $9.7 \%$ & 42 & $10.3 \%$ \\
60 to 75 & 2168 & $18.2 \%$ & 81 & $19.9 \%$
\end{tabular}


A

$A B$

B

O

$45.5 \%$

198

$48.6 \%$

423

$3.6 \%$

16

$3.9 \%$

919

$7.7 \%$

22

$5.4 \%$

161

$5151 \quad 43.3 \%$

171

$42.0 \%$

1621,142 donations from year 2019 were analyzed) but none of them were 163 positive when testing separately $\lg G$, $\lg A$ or $\lg M$. Their ODs were 1.05, 1641.08 and 5.46 respectively.

165 Seropositivity rate (positive donations x 100 / total analyzed donations per 166 week) grew up from week 11 to week 25 , reaching a $11.1 \%$ peak, with just 167 subtle descents until week 43 when the second wave arose in our country. 168 Plateau was reached by the last week of May 2020, 11 weeks after close 169 lockdown in Spain and 17 weeks after 31/01, the date of the first declared 170 case in or country. 660 out of 9,886 single donors were positive within 171 weeks 11 to 43 (Fig 1). Seroprevalence was therefore $6.73 \%$ at the end of 172 the first 
173 wave.

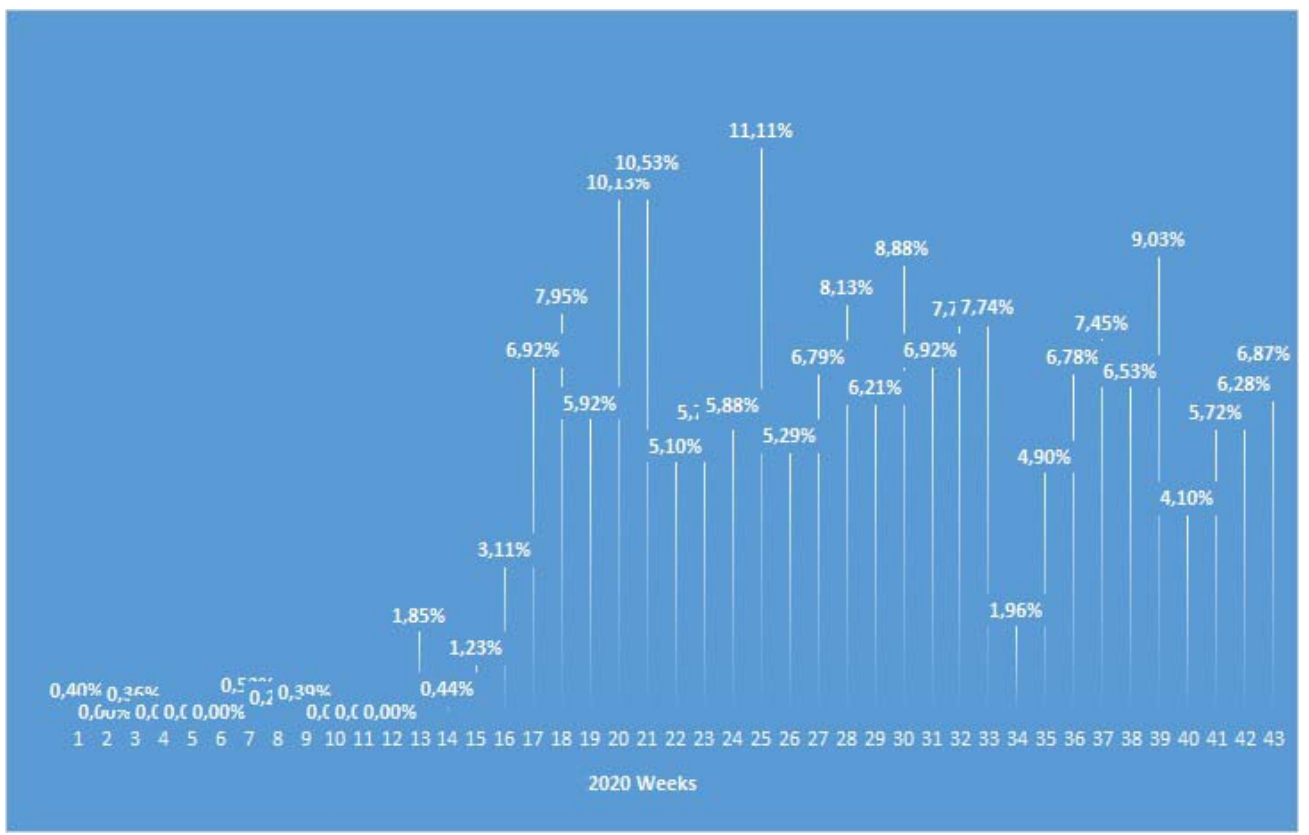

175 Fig 1. COVID-19 seropositive donation rate. 2020 evolution by weeks.

Immunoglobulin levels didn't show any differences as comparing positive and negative donations (Table 3). Haemoglobin (14.9 vs $14.7 \mathrm{~g} / \mathrm{dL} ; \mathrm{p}<0.001)$ and haematocrit $(45.2 \%$ vs $44.4 \% ; p<0.001)$ were both significantly lower in positive donations. Leukocyte count (WBC) was as well slightly lower (7.23 vs 7.06 cells ${ }^{*} 10^{3} / \mu \mathrm{L} ; \mathrm{p}=0.026$ ). Trends were found of lower number of neutrophils (4.3 vs. 4.2 cells ${ }^{*} 10^{3} / \mu \mathrm{L} ; \mathrm{p}=0.117$ ), lower count of monocytes ( 0.46 vs. 0.45 cells $^{*} 10^{3} / \mu \mathrm{L}$; $\mathrm{p}=0.117)$ and higher percent of basophils $(0.49 \%$ vs $0.51 \% ; \mathrm{p}=0.131)$ in positive donations as compared with negative ones.

Table 3. Descriptive analyses of age, immunoglobulins and haemogram of 
reactive and non-reactive donations

\begin{tabular}{|c|c|c|c|c|c|c|}
\hline & & $\mathrm{n}$ & mean & median & SD & IQR \\
\hline \multirow[t]{2}{*}{ Age } & Negative & 11906 & 44.0 & 45.8 & 12.8 & $34.2-54.2$ \\
\hline & Positive & 407 & 44.2 & 46.5 & 13.0 & $32.8-54.8$ \\
\hline \multirow[t]{2}{*}{$\lg A$} & Negative & 194 & 236.9 & 223.5 & 101.3 & $163-292$ \\
\hline & Positive & 5 & 224.4 & 274.0 & 92.3 & $134-282$ \\
\hline \multirow[t]{2}{*}{$\lg G$} & Negative & 194 & 1065.4 & 1037.5 & 217.4 & $910-1185$ \\
\hline & Positive & 5 & 1123.8 & 1095.0 & 249.9 & $924-1229$ \\
\hline \multirow[t]{2}{*}{$\lg M$} & Negative & 194 & 113.3 & 100.5 & 64.8 & $69-138$ \\
\hline & Positive & 5 & 103.0 & 89.0 & 41.7 & $78-97$ \\
\hline \multirow[t]{2}{*}{$\mathrm{WBC}^{\mathrm{a}}$} & Negative & 9759 & 7.4 & 7.2 & 1.8 & $6.13-8.49$ \\
\hline & Positive & 346 & 7.2 & 7.1 & 1.6 & $6.11-8.12$ \\
\hline \multirow[t]{2}{*}{$\mathrm{Hb}^{\mathrm{b}}$} & Negative & 11218 & 14.9 & 14.9 & 1.3 & 14-15.8 \\
\hline & Positive & 386 & 14.7 & 14.7 & 1.2 & $13.8-15.4$ \\
\hline \multirow[t]{2}{*}{ Hematocrite $^{b}$} & Negative & 11218 & 45.1 & 45.2 & 3.5 & $42.7-47.6$ \\
\hline & Positive & 386 & 44.5 & 44.4 & 3.5 & $42.3-46.6$ \\
\hline \multirow[t]{2}{*}{ Platelets } & Negative & 11217 & 247.9 & 243.0 & 54.3 & $211-280$ \\
\hline & Positive & 386 & 247.3 & 242.5 & 55.7 & $206-276$ \\
\hline
\end{tabular}

Neutrophil

$\begin{array}{lrrrrrr}\text { count }^{\mathrm{c}} & \text { Negative } & 11217 & 4.4 & \mathbf{4 . 3} & 1.4 & 3.44-5.19 \\ & \text { Positive } & 386 & 4.3 & \mathbf{4 . 2} & 1.2 & 3.34-5.03 \\ \text { Neutrophil \% } & \text { Negative } & 11217 & 58.8 & \mathbf{5 8 . 9} & 7.5 & 53.8-63.9 \\ & \text { Positive } & 386 & 58.5 & \mathbf{5 8 . 7} & 7.1 & 54.3-63.4\end{array}$


Lymphocyte

$\begin{array}{lcccccc}\text { count } & \text { Negative } & 11216 & 2.3 & \mathbf{2 . 3} & 0.7 & 1.86-2.72 \\ & \text { Positive } & 386 & 2.3 & \mathbf{2 . 2} & 0.6 & 1.89-2.64 \\ \text { Lymphocyte \% } & \text { Negative } & 11216 & 31.7 & \mathbf{3 1 . 5} & 6.9 & 27-36.2 \\ & \text { Positive } & 386 & 32.1 & \mathbf{3 2 . 4} & 6.6 & 27.3-36.1\end{array}$

Monocyte

count

Negative 11216

0.5

0.46

0.1

$0.38-0.56$

Positive

386

0.5

0.46

0.1

$0.37-0.54$

Monocyte \%

Negative 11216

6.5

6.4

1.5

5.5-7.4

Positive $\quad 386$

6.5

6.4

1.4

5.5-7.3

Eosinophil

count

Negative 11217

0.2

0.2

0.1

0.09-0.23

Positive

386

0.2

0.1

$0.1 \quad 0.09-0.22$

Eosinophil \%

Negative 11217

Positive

386

2.4

2.1

1.8

1.3-3.2

Basophil count

Negative

11217

0.0

2.0

1.7

1.3-3.1

Positive

386

0.0

0.0

0.0

$0.02-0.04$

$\begin{array}{ccccccc} & \text { Positive } & 386 & 0.0 & \mathbf{0 . 0} & 0.0 & 0.02-0.04 \\ \text { Basophil \% } & \text { Negative } & 11217 & 0.5 & \mathbf{0 . 5} & 0.2 & 0.3-0.6 \\ & \text { Positive } & 386 & 0.5 & \mathbf{0 . 5} & 0.3 & 0.4-0.6\end{array}$

178 Units: Immunoglobulins (mg/dL); Hb (g/dL); haemogram counts

179 (cells* $\left.10^{3} / \mu \mathrm{L}\right)$

$180{ }^{a} p=0.026$

$181 \quad{ }^{b} p<0.001$

$182{ }^{\mathrm{c}} \mathrm{p}=0.117$ 
$183{ }^{d} p=0.131$

184 As layering data by sex (Table 4$)$, the fact arose that haematocrit $(46.8 \%$

185 vs 45.9\%; $p<0.001$ ) and haemoglobin (15.5 vs $15.3 \mathrm{~g} / \mathrm{dL} ; \mathrm{p}<0.001$ ) were

186 significantly lower just in males and that positive females had significantly

187 less WBC (7.67 vs 7.25 cells* $10^{3} / \mu \mathrm{L} ; \mathrm{p}=0.027$ ) and neutrophil numbers

188 (4.53 vs 4.27 cells* $\left.10^{3} / \mu L ; p=0.014\right)$.

189 Table 4. Descriptive analysis of age, age, immunoglobulins and

190 haemogram of reactive and non-reactive donations layered by sex

\begin{tabular}{|c|c|c|c|c|c|c|c|}
\hline & & & $\mathbf{n}$ & mean & median & SD & IQR \\
\hline \multirow[t]{4}{*}{ Age } & Female & Negative & 4531 & 42.0 & 43.50 & 13.3 & $30.3-53.3$ \\
\hline & & Positive & 164 & 42.1 & 45.39 & 13.6 & 28.6-53.1 \\
\hline & Male & Negative & 7375 & 45.2 & 46.81 & 12.2 & $36.7-54.8$ \\
\hline & & Positive & 243 & 45.7 & 46.98 & 12.5 & $35.5-55.9$ \\
\hline \multirow[t]{4}{*}{$\lg A$} & Female & Negative & 54 & 214.3 & 200.00 & 97.0 & $157-250$ \\
\hline & & Positive & 3 & 235.3 & 274.00 & 105.5 & $116-316$ \\
\hline & Male & Negative & 140 & 245.6 & 233.50 & 101.9 & $176.5-302.5$ \\
\hline & & Positive & 2 & 208.0 & 208.00 & 104.7 & $134-282$ \\
\hline \multirow[t]{4}{*}{$\lg G$} & Female & Negative & 54 & 1076.4 & 1062.50 & 191.3 & $964-1186$ \\
\hline & & Positive & 3 & 1082.7 & 1095.00 & 152.9 & $924-1229$ \\
\hline & Male & Negative & 140 & 1061.2 & 1029.00 & 227.2 & $903-1181.5$ \\
\hline & & Positive & 2 & 1185.5 & 1185.50 & 436.3 & 877-1494 \\
\hline \multirow[t]{3}{*}{$\lg M$} & Female & Negative & 54 & 143.1 & 129.50 & 64.6 & 94-169 \\
\hline & & Positive & 3 & 114.3 & 89.00 & 53.7 & $78-176$ \\
\hline & Male & Negative & 140 & 101.8 & 90.00 & 61.3 & $64.5-121$ \\
\hline
\end{tabular}




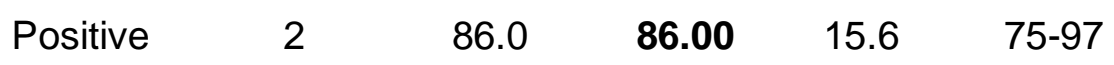

Mean

corpuscular

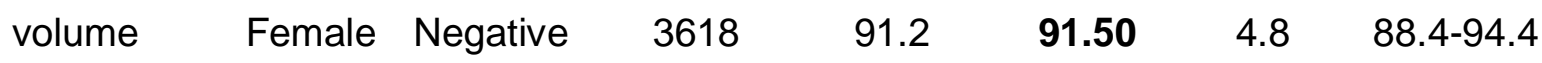

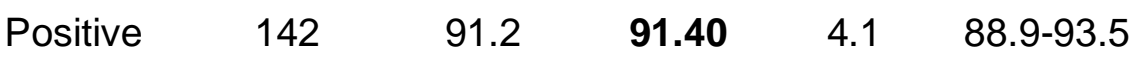

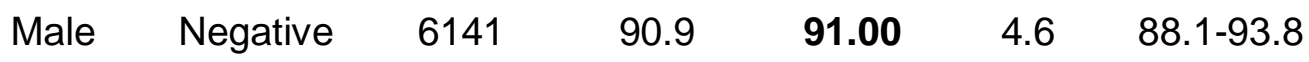

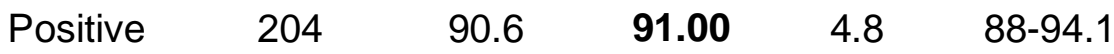

$\begin{array}{lllllll}\text { WBC } & \text { Female }^{\mathrm{a}} \text { Negative } & 3618 & 7.8 & 7.69 & 1.9 & 6.49-9\end{array}$

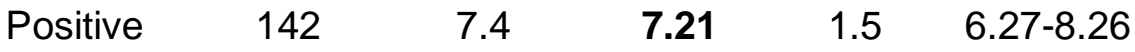

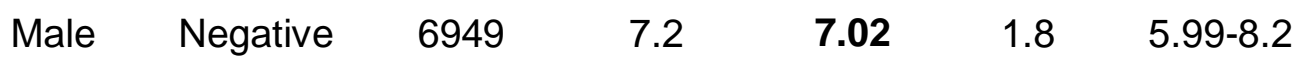

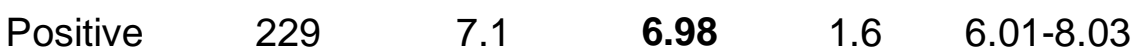

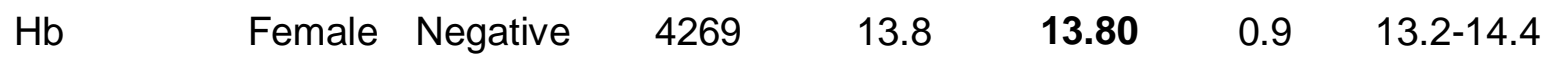

$\begin{array}{llllll}\text { Positive } & 157 & 13.7 & \mathbf{1 3 . 8 0} & 0.9 & \mathbf{1 3 . 2}-14.3\end{array}$

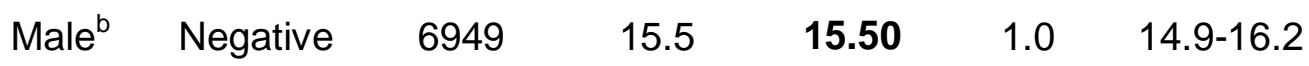

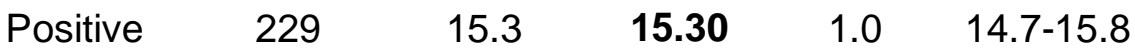

$\begin{array}{llllll}\text { Haematocrit Female } & \text { Negative } & 4269 & 42.4 & \mathbf{4 2 . 3 0} & 2.7\end{array}$

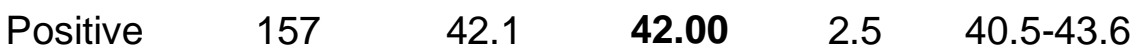

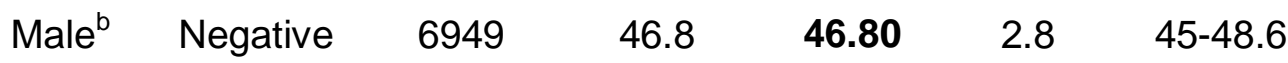

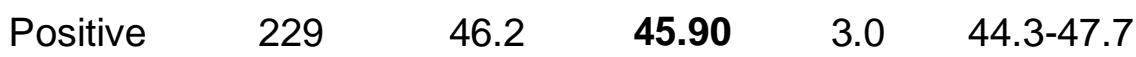

$\begin{array}{lllllll}\text { Platelets } & \text { Female }^{c} \text { Negative } & 4268 & 265.9 & \mathbf{2 6 1 . 0 0} & 56.6 & 228-300\end{array}$

$\begin{array}{rrrrrrr} & \text { Positive } & 157 & 261.0 & \mathbf{2 5 5 . 0 0} & 60.5 & 219-296 \\ \text { Male } & \text { Negative } & 6949 & 236.8 & \mathbf{2 3 3 . 0 0} & 49.6 & 204-266 \\ & \text { Positive } & 229 & 237.9 & \mathbf{2 3 4 . 0 0} & 50.2 & 203-266\end{array}$

Neutrophil

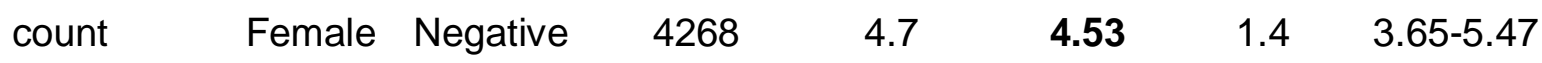




$\begin{array}{ccccccc} & \text { Positive } & 157 & 4.4 & \mathbf{4 . 2 7} & 1.2 & 3.56-5.08 \\ \text { Male } & \text { Negative } & 6949 & 4.3 & \mathbf{4 . 1 0} & 1.4 & 3.34-4.95 \\ & \text { Positive } & 229 & 4.2 & \mathbf{4 . 1 0} & 1.2 & 3.24-4.97\end{array}$

Neutrophil

$\%$

$\begin{array}{ccccccc}\text { Female } & \text { Negative } & 4268 & 59.2 & \mathbf{5 9 . 4 0} & 7.4 & 54.2-64.3 \\ & \text { Positive } & 157 & 58.1 & \mathbf{5 8 . 1 0} & 7.2 & 54-62.9 \\ \text { Male } & \text { Negative } & 6949 & 58.6 & \mathbf{5 8 . 7 0} & 7.5 & 53.6-63.6 \\ & \text { Positive } & 229 & 58.8 & \mathbf{5 8 . 7 0} & 7.0 & 54.6-63.5\end{array}$

Lymphocyte

\begin{tabular}{|c|c|c|c|c|c|c|c|}
\hline \multirow{2}{*}{\multicolumn{2}{|c|}{ Female }} & Negative & 4267 & 2.5 & 2.39 & 0.7 & $1.97-2.88$ \\
\hline & & Positive & 157 & 2.4 & 2.35 & 0.6 & $2.02-2.81$ \\
\hline & Male & Negative & 6949 & 2.2 & 2.18 & 0.6 & $1.8-2.6$ \\
\hline & & Positive & 229 & 2.2 & 2.17 & 0.6 & $1.8-2.5$ \\
\hline
\end{tabular}

Lymphocyte

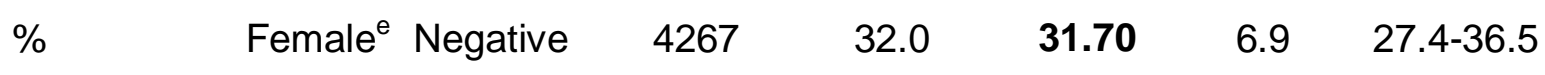

\begin{tabular}{|c|c|}
\hline Positive & 157 \\
\hline
\end{tabular}

$\begin{array}{llllll}\text { Male } \quad \text { Negative } & 6949 & 31.5 & 31.30 & 6.9 & 26.8-36.1\end{array}$

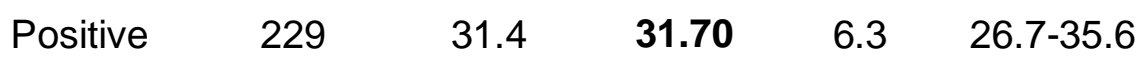

Monocyte

\begin{tabular}{|c|c|c|c|c|c|c|c|}
\hline \multirow{2}{*}{\multicolumn{2}{|c|}{ Female ${ }^{f}$}} & Negative & 4267 & 0.5 & 0.46 & 0.1 & $0.37-0.55$ \\
\hline & & Positive & 157 & 0.5 & 0.44 & 0.1 & $0.36-0.52$ \\
\hline & Male & Negative & 6949 & 0.5 & 0.46 & 0.1 & $0.38-0.5$ \\
\hline & & Positive & 229 & 0.5 & 0.47 & 0.1 & $0.38-0.5$ \\
\hline
\end{tabular}

Monocyte

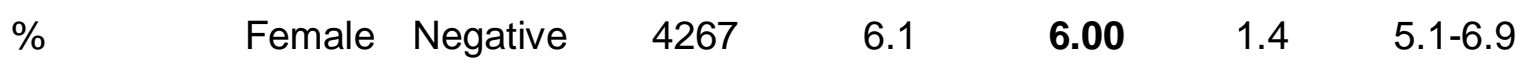




$\begin{array}{ccccccc} & \text { Positive } & 157 & 6.1 & 6.00 & 1.4 & 5.2-6.9 \\ \text { Male } & \text { Negative } & 6949 & 6.7 & 6.60 & 1.5 & 5.7-7.6 \\ & & & & & & \\ & \text { Positive } & 229 & 6.7 & 6.70 & 1.4 & 5.8-7.6\end{array}$

Eosinophil

count

Female

Negative $\quad 4268$

0.2

0.14

$0.1 \quad 0.09-0.21$

Positive

157

0.2

0.13

0.1

0.09-0.19

Male Negative 6949

0.2

0.16

0.1

$0.1-0.24$

Positive 229

0.2

0.15

$0.1 \quad 0.09-0.23$

Eosinophil

\begin{tabular}{|c|c|c|c|c|c|c|c|}
\hline \multirow{2}{*}{\multicolumn{2}{|c|}{ Female }} & Negative & 4268 & 2.2 & 1.80 & 1.6 & $1.2-2.8$ \\
\hline & & Positive & 157 & 2.3 & 1.80 & 1.7 & $1.1-2.8$ \\
\hline & \multirow[t]{2}{*}{ Male } & Negative & 6949 & 2.7 & 2.20 & 1.8 & $1.4-3.4$ \\
\hline & & Positive & 229 & 2.5 & 2.10 & 1.7 & $1.4-3.2$ \\
\hline
\end{tabular}

Basophil

\begin{tabular}{|c|c|c|c|c|c|c|c|}
\hline \multirow[t]{4}{*}{ count } & Female & Negative & 4268 & 0.0 & 0.03 & 0.0 & $0.02-0.05$ \\
\hline & & Positive & 157 & 0.0 & 0.04 & 0.0 & $0.02-0.05$ \\
\hline & Male & Negative & 6949 & 0.0 & 0.03 & 0.0 & $0.02-0.04$ \\
\hline & & Positive & 229 & 0.0 & 0.03 & 0.0 & $0.02-0.04$ \\
\hline \multirow[t]{4}{*}{ Basophil \% } & Female $^{g}$ & Negative & 4268 & 0.5 & 0.40 & 0.2 & $0.3-0.6$ \\
\hline & & Positive & 157 & 0.5 & 0.50 & 0.3 & $0.3-0.6$ \\
\hline & Male & Negative & 6949 & 0.5 & 0.50 & 0.2 & $0.3-0.6$ \\
\hline & & Positive & 229 & 0.5 & 0.50 & 0.2 & $0.4-0.6$ \\
\hline
\end{tabular}

192 Units:immunoglobulins (mg/dL); $\mathrm{Hb}(\mathrm{g} / \mathrm{dL})$; haemogram counts

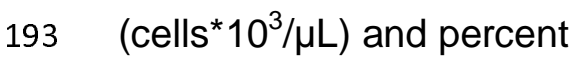



$194{ }^{a} p=0.027$
$195{ }^{b} p<0.001$
$196{ }^{c} p=0.152$
$197{ }^{d} p=0.065$
$198 \mathrm{e}^{\mathrm{e}} \mathrm{p}=0.074$
$199{ }^{f} p=0.132$
$200{ }^{g} p=0.060$
201
202
203 Some trends were found as well related to sex. Regarding positive women
204 neutrophil percent would be lower (59.4\% vs 58.10\% $p=0.065)$,
205 lymphocyte percent, higher (31.7\% vs 33.10\%; $\mathrm{p}=0.074)$, platelet count,
206 (261 vs 255 cells $\left.^{*} 10^{3} / \mu \mathrm{L} ; \mathrm{p}=0.152\right)$, monocyte count (0.46 vs 0.44
207 cells $\left.{ }^{*} 10^{3} / \mu \mathrm{L} ; \mathrm{p}=0.132\right)$ and basophil percent $(0.5 \%$ vs $0.4 \% ; p=0.060)$
208 lower. Anaemia was more frequent in positive male donors as $(6.1 \% \mathrm{vs}$
$2099.6 \% ; p=0.031)$. No other significant differences were found either in
210 males, females or overall (Fig 2). 


\begin{tabular}{|c|c|c|c|}
\hline & $\begin{array}{l}\overline{\bar{\pi}} \\
\overline{0} \\
\text { ठे }\end{array}$ & $\frac{\frac{\omega}{\pi}}{\Sigma}$ & $\begin{array}{l}\frac{\nu}{\tilde{N}} \\
\frac{\sum_{\nu}}{4}\end{array}$ \\
\hline $\mathrm{Hb}$ & $\downarrow$ & $\downarrow$ & - \\
\hline Hematocrite & $\downarrow$ & $\downarrow$ & - \\
\hline Leukocyte count & $\downarrow$ & - & $\downarrow$ \\
\hline Platelet count & - & - & $\Downarrow$ \\
\hline Neutrophil count & $\downarrow$ & - & $\downarrow$ \\
\hline Neutrophil \% & - & - & $\uparrow$ \\
\hline Lymphocyte count & - & - & $\Downarrow$ \\
\hline Lymphocyte \% & - & - & $\downarrow$ \\
\hline Monocyte count & $\downarrow$ & - & $\Downarrow$ \\
\hline Basophil \% & $\uparrow$ & - & $\Downarrow$ \\
\hline
\end{tabular}

212 Fig 2. Summary of haematological features in SARS-CoV-2 positive

donations as compared to negative ones. Intense-coloured arrows

214 represent significant differences. Light-coloured arrows represent 215 statistical trends.

\section{Discussion}

218 A similar seroprevalence to that reported in general population was found 219 among blood donors without COVID-19 antecedents. The $6.73 \%$ 220 seroprevalence is a little lower than that reported in the seroprevalence 221 study ENE-COVID-19 promoted by the Instituto Nacional the 222 Epidemiología [11], where it is reported that a $7.2 \%$ of the participants in 223 our region would have anti SARS-CoV2 IgG antibodies by the end of June 224 2020. Almost one quarter people in our region (25.51\% according to 225 National Institute of Statistics, INE 2020 data) is older than 65 and elderly 226 are necessarily underrepresented in this study. That happens as well with 
medRxiv preprint doi: https://doi.org/10.1101/2021.04.28.21256242; this version posted June 24, 2021. The copyright holder for this preprint (which was not certified by peer review) is the author/funder, who has granted medRxiv a license to display the preprint in perpetuity.

It is made available under a CC-BY 4.0 International license .

227 population aged under $18(<4.5 \%$ in our region). One limitation of this 228 study is this of restricted age, but it would make just a little difference of $2290.5 \%$ in seroprevalence estimation. Immunization rate would be a bit 230 higher regarding the fact that some individuals who do not exhibit 231 antibodies but still mount an efficient specific T-cell response [2]

232 Perhaps the main strength of our study is the large number of samples we 233 randomized and the long period we tested (15 months). The first recorded 234 COVID-19 case in Spain was reported on 31/01/2020, but three positive 235 donations were found as testing samples collected along 2019. That could 236 be meaningful in two opposite ways: one possible explanation would be 237 cross reactivity to seasonal cold coronaviruses and the other would be that 238 SARS-CoV-2 might have been circulating at least since 2019 summer.

239 It is now known that up to $28 \%$ people would have protection against

240 SARS-CoV-2 due to cross reaction to other coronaviruses [2]. It has been 241 checked that long-term cross-reactive both T-cells and antibodies can be 242 correlates of protection against COVID-19 [12]. Asymptomatic or mild 243 cases couldn't be diagnosed or reported before the first tests were 244 available. There are other reports about SARS-CoV-2 circulating in 245 Europe along 2019 [13]. Our three 2019 positive donors would be even 246 earlier cases, but the simplest explanation would be that of cross247 reactions. Some of the positives along the period of study might be as well 248 cross-reactive or both reactive due to SARS-CoV-2 infection together with 249 previous coronavirus immunity. 
medRxiv preprint doi: https://doi.org/10.1101/2021.04.28.21256242; this version posted June 24, 2021. The copyright holder for this preprint (which was not certified by peer review) is the author/funder, who has granted medRxiv a license to display the preprint in perpetuity.

It is made available under a CC-BY 4.0 International license.

250 Conversely to clinical forms of COVID-19 [14,15] neither age nor sex have

251 an influence on the probability to develop an asymptomatic COVID-19

252 infection in our cohort. Several smaller seroprevalence reports from

253 European countries support this feature [16-20]. Age and sex would not

254 therefore have a role in SARSCoV-2 infection susceptibility, but only in

255 their progression to severe forms.

256 Blood group has been reported elsewhere [7,21-23] to confere

257 susceptibility or determine severity of SARS-CoV2 infection. Most

258 published studies do not separate them. It should be noticed that there are

259 no large studies characterizing asymptomatic cases in literature, perhaps

260 this is one of the reports comprising one of the largest asymptomatic

261 cohorts to date. Relationship of $A B O$ system with susceptibility is not

262 supported by our data. We excluded convalescent plasma donors,

263 opposite to other published studies, that focused into these asymptomatic

264 positive donors [22]. Perhaps blood group might play a role only in

265 symptomatic cases and would be therefore related to severity but not to

266 asymptomatic SARS-CoV-2 infection. Another surface antigens such as

267 HLA or KIR might be as well involved and should be studied.

268 Several blood count anomalies have been reported in SARS-CoV2

269 infection, notably lymphopenia and neutrophilia are related to the severest

270 forms [24]. Our data conversely reveal that asymptomatic positive women

271 had a significant lower count of WBC and neutrophils as compared to

272 negative ones. Similar low counts of WBC and neutrophils have been

273 reported in young $(<19)$ cohorts, comprising mainly mild and asymptomatic 
medRxiv preprint doi: https://doi.org/10.1101/2021.04.28.21256242; this version posted June 24, 2021. The copyright holder for this preprint (which was not certified by peer review) is the author/funder, who has granted medRxiv a license to display the preprint in perpetuity.

It is made available under a CC-BY 4.0 International license .

274 cases [25]. Perhaps this feature might be therefore a correlate of

275 mildness. Elevations of monocytes have been elsewhere reported in short

276 series of mild cases [26]. Our observation will be in the same line of those

277 findings but a gender bias cannot be discarded so far seropositive women

278 would go in the opposite direction.

279 Several reports about haemolytic anaemia secondary to SARSCoV-2 have

280 been published [27-30]. We cannot discard a mild silent haemolytic

281 anaemia in asymptomatic cases but just small asymptomatic cohorts are

282 published to date.

283 We can conclude that seroprevalence estimations through blood donation

284 analysis mirror population-based surveys. Sex and age would not affect

285 COVID-19 susceptibility but its severity. Gender differences are present

286 even in asymptomatic individuals: positive females have lower lymphocyte

287 counts whereas positive males, present anaemia more frequently than

288 negative ones. Further studies in large cohorts are needed to confirm

289 these gender differences and to characterize asymptomatic COVID-19

290 cases as they can help better understand immune response to COVID-19,

291 its pathogenesis and prognosis.

\section{Acknowledgements}

LB and MCM conceived and designed the study, NH, NO; MP and AP acquired data, IP, AJ and MIGF performed analysis and interpretation of laboratory data, MCM and LB drafted the article and revised it critically for 
medRxiv preprint doi: https://doi.org/10.1101/2021.04.28.21256242; this version posted June 24, 2021. The copyright holder for this preprint (which was not certified by peer review) is the author/funder, who has granted medRxiv a license to display the preprint in perpetuity.

It is made available under a CC-BY 4.0 International license.

important intellectual content, all authors provided final approval of the version to be submitted.

The authors thank to all blood donors for making this work possible by allowing research use of their samples by the Biobanco del Centro de Hemoterapia y Hemodonación de Castilla y León. They also thank the staff in charge of blood donation, and lab technicians for their efforts and Roche for its support. 
medRxiv preprint doi: https://doi.org/10.1101/2021.04.28.21256242; this version posted June 24, 2021. The copyright holder for this preprint (which was not certified by peer review) is the author/funder, who has granted medRxiv a license to display the preprint in perpetuity.

It is made available under a CC-BY 4.0 International license.

\section{References}

1. Busch MP, Stone M. Serosurveillance for Severe Acute Respiratory Syndrome Coronavirus 2 (SARS-CoV-2) Incidence Using Global Blood Donor Populations. Clin Infect Dis. 2020;72(2):254-256.

2. Sekine T, Perez-Potti A, Rivera-Ballesteros O, Strålin K, Gorin JB, Olsson A, et al. Robust $T$ Cell Immunity in Convalescent Individuals with Asymptomatic or Mild COVID-19. Cell. 2020 Oct 1;183(1):158-168.e14. doi: 10.1016/j.cell.2020.08.017. Epub 2020 Aug 14. PMID: 32979941; PMCID: PMC7427556.

3. Wouters $E$, Steenhuis $M$, Schrezenmeier $H$, Tiberghien $P$, Harvala $H$, Feys $\mathrm{HB}$, et al. Evaluation of SARS-CoV-2 antibody titers and potency for convalescent plasma donation: a brief commentary. Vox Sang. 2021 May;116(5):493-496. doi: 10.1111/vox.13060. Epub 2020 Dec 23. PMID: 33368373.

4. Angulo FJ, Finelli L, Swerdlow DL. Estimation of US SARS-CoV-2 Infections, Symptomatic Infections, Hospitalizations, and Deaths Using Seroprevalence Surveys. JAMA Netw Open. 2021;4(1):e2033706.

5. Friston K, Costello A, Pillay D. 'Dark matter', second waves and epidemiological modelling. BMJ Glob Health. 2020;5(12):e003978.

6. Netea MG, van der Meer JW, van Crevel R. BCG vaccination in health care providers and the protection against COVID-19.J Clin Invest. 2021;131(2):e145545 .

7. Gallais F, Velay A, Nazon C, Wendling MJ, Partisani M, Sibilia J, et al. Intrafamilial Exposure to SARS-CoV-2 Associated with Cellular Immune Response without Seroconversion, France. Emerg Infect Dis. 2021;27(1):113-21

8. Gallian P, Pastorino B, Morel P, Chiaroni J, Ninove L, de Lamballerie X. Lower prevalence of antibodies neutralizing SARS-CoV-2 in group $O$ French blood donors. Antiviral Res. 2020;181:104880

9. Kohmer N, Westhaus $S$, Rühl C, Ciesek $S$, Rabenau HF. Brief clinical evaluation of six high-throughput SARS-CoV-2 IgG antibody assays. J Clin Virol. 2020;129:104480

10. Müller L, Ostermann PN, Walker A, Wienemann T, Mertens A, Adams O, et al. Sensitivity of anti-SARS-CoV-2 serological assays in a high-prevalence setting. Eur J Clin Microbiol Infect Dis. 2021 May;40(5):1063-1071. doi: 10.1007/s10096-021-04169-7. Epub 2021 Feb 3. PMID: 33534090; PMCID: PMC7856849.

11. Pastor-Barriuso R, Pérez-Gómez $B$, Hernán MA, Pérez-Olmeda $M$, Yotti $R$, Oteo-Iglesias J, et al. Infection fatality risk for SARS-CoV-2 in community dwelling population of Spain: nationwide seroepidemiological study. BMJ. 
medRxiv preprint doi: https://doi.org/10.1101/2021.04.28.21256242; this version posted June 24, 2021. The copyright holder for this preprint (which was not certified by peer review) is the author/funder, who has granted medRxiv a license to display the preprint in perpetuity.

It is made available under a CC-BY 4.0 International license.

2020 Nov 27;371:m4509. doi: 10.1136/bmj.m4509. PMID: 33246972; PMCID: PMC7690290.

12. Poonia B, Kottilil S. Immune Correlates of COVID-19 Control. Front Immunol. 2020;11:569611

13. Murtas R, Decarli A, Russo AG. Trend of pneumonia diagnosis in emergency departments as a COVID-19 surveillance system: a time series study. BMJ Open. 2021;11(2):e044388.

14. Jurado A, Martín MC, Abad-Molina C, et al. COVID-19: age, Interleukin-6, C-reactive protein, and lymphocytes as key clues from a multicentre retrospective study. Immun Ageing. 2020;17:22

15. Martín MC, Jurado A, Abad-Molina $C$, Orduña $A$, Yarce $O$, Navas AM, et al. The age again in the eye of the COVID-19 storm: evidence-based decision making. Immun Ageing. 2021 May 20;18(1):24. doi: 10.1186/s12979-02100237-w. PMID: 34016150; PMCID: PMC8134808.

16. Dickson E, Palmateer NE, Murray J, Robertson C, Waugh C, Wallace LA, et al. Enhanced surveillance of COVID-19 in Scotland: population-based seroprevalence surveillance for SARS-CoV-2 during the first wave of the epidemic. Public Health. 2021 Jan;190:132-134. doi: 10.1016/j.puhe.2020.11.014. Epub 2020 Nov 24. PMID: 33453689; PMCID: PMC7685039.

17. Hallal PC, Hartwig FP, Horta BL, Silveira MF, Struchiner CJ, Vidaletti LP, et al. SARS-CoV-2 antibody prevalence in Brazil: results from two successive nationwide serological household surveys. Lancet Glob Health. 2020 Nov;8(11):e1390-e1398. doi: 10.1016/S2214-109X(20)30387-9. Epub 2020 Sep 23. PMID: 32979314; PMCID: PMC7511212.

18. Bogogiannidou Z, Speletas M, Vontas A, Nikoulis DJ, Dadouli K, Kyritsi MA, et al. Repeated Leftover Serosurvey of SARS-CoV-2 IgG Antibodies in Greece, May to August 2020. Vaccines (Basel). 2021 May 13;9(5):504. doi: 10.3390/vaccines9050504. PMID: 34068100; PMCID: PMC8152765.

19. Biggs HM, Harris JB, Breakwell L, Dahlgren FS, Abedi GR, Szablewski CM, et al. Seroprevalence of anti-SARS-CoV-2 IgG antibodies in Geneva, Switzerland (SEROCOV-POP): a population-based study. Lancet. 2020;396(10247):313-319

20. Pendu JL, Breiman A, Rocher J, Dion $M$, Ruvoën-Clouet N. ABO Blood Types and COVID-19: Spurious, Anecdotal, or Truly Important Relationships? A Reasoned Review of Available Data. Viruses. 2021;13(2):160

21. Muñiz-Diaz E, Llopis J, Parra R, Roig I, Ferrer G, Grifols J, et al. Relationship between the ABO blood group and COVID-19 susceptibility, severity and mortality in two cohorts of patients. Blood Transfus. 2021 Jan;19(1):54-63. doi: 10.2450/2020.0256-20. Epub 2020 Nov 12. PMID: 33196417; PMCID: PMC7850930. 
medRxiv preprint doi: https://doi.org/10.1101/2021.04.28.21256242; this version posted June 24, 2021. The copyright holder for this preprint (which was not certified by peer review) is the author/funder, who has granted medRxiv a license to display the preprint in perpetuity.

It is made available under a CC-BY 4.0 International license .

22. Zalba Marcos S, Antelo ML, Galbete A, Etayo M, Ongay E, García-Erce JA. Infection and thrombosis associated with COVID-19: Possible role of the ABO blood group. Infección y trombosis asociada a la COVID-19: posible papel del grupo sanguíneo ABO. Med Clin (Barc). 2020;155(8):340-343. doi:10.1016/j.medcli.2020.06.020

23. Agbuduwe C, Basu S. Haematological manifestations of COVID-19: From cytopenia to coagulopathy. Eur J Haematol. 2020;105(5):540-546

24. Toba N, Gupta S, Ali AY, ElSaban M, Khamis AH, Ho SB, Popatia R. COVID-19 under 19: A meta-analysis. Pediatr Pulmonol. 2021 Jun;56(6):1332-1341. doi: 10.1002/ppul.25312. Epub 2021 Feb 25. PMID: 33631060; PMCID: PMC8013606

25. Gatti A, Radrizzani D, Viganò P, Mazzone A, Brando B. Decrease of Non-Classical and Intermediate Monocyte Subsets in Severe Acute SARS-CoV-2 Infection. Cytometry A. 2020 Sep;97(9):887-890. doi: 10.1002/cyto.a.24188. Epub 2020 Aug 14. PMID: 32654350; PMCID: PMC7404377

26. Gruden G, Beggiato E, Camerino E, Capriotti S, Canepa S, Scandella M, et al. Treatment with eltrombopag of severe immune thrombocytopenia and hemolytic anemia associated with COVID-19 pneumonia: a case report. Ther Adv Hematol. 2021 Apr 27;12:20406207211011353. doi: 10.1177/20406207211011353. PMID: 33995989; PMCID: PMC8082984.

27. Liput JR, Jordan K, Patadia R, Kander E. Warm Autoimmune Hemolytic Anemia Associated With Asymptomatic SARS-CoV-2 Infection. Cureus. 2021 Mar 25;13(3):e14101. doi: 10.7759/cureus.14101. PMID: 33927918; PMCID: PMC8075761.

28. Georgy JT, Jayakaran JAJ, Jacob AS, Gunasekaran K, Korula PJ, Devasia AJ, et al. Evans syndrome and immune thrombocytopenia in two patients with COVID-19. J Med Virol. 2021 May;93(5):2642-2644. doi: 10.1002/jmv.26906. Epub 2021 Mar 9. PMID: 33634495; PMCID: PMC8013600.

29. Mausoleo A, Henriquez S, Goujard C, Roque-Afonso AM, Noel N, Lambotte O. Severe IgA-mediated autoimmune hemolytic anemia triggered by SARS-CoV-2 infection. Leuk Lymphoma. 2021 Feb 22:1-5. doi: 10.1080/10428194.2021.1888378. Epub ahead of print. PMID: 33618609.

30. Goud PT, Bai D, Abu-Soud HM. A Multiple-Hit Hypothesis Involving Reactive Oxygen Species and Myeloperoxidase Explains Clinical Deterioration and Fatality in COVID-19. Int J Biol Sci. 2021 Jan 1;17(1):6272

31. Dattilo M. The role of host defences in Covid 19 and treatments thereof. Mol Med. 2020;26(1):90 
medRxiv preprint doi: https://doi.org/10.1101/2021.04.28.21256242; this version posted June 24, 2021. The copyright holder for this preprint (which was not certified by peer review) is the author/funder, who has granted medRxiv a license to display the preprint in perpetuity.

It is made available under a CC-BY 4.0 International license.

296

297 Abbreviations

298 Immunoglobulin G (lgG); immunoglobulin A ( $\lg A)$; immunoglobulin M

299 (IgM); polymerase chain reaction (PCR); standard deviation (SD);

300 interquartile range (IQR); White blood cells (WBC), haemoglobin ( $\mathrm{Hb})$,

301 haematocrit (HCT)

302

303 